\title{
HSP70 expression before and after treatment and its clinical value in patients with acute angle-closure glaucoma
}

\author{
HONG CHEN $^{1}$, AIJUN TIAN ${ }^{1}$, YIXIANG WU ${ }^{2}$, RONGRONG LI ${ }^{1}$, \\ RUIJUAN HAN ${ }^{1}$, XIAOWEI XU ${ }^{3}$ and SUMIAN CHENG ${ }^{1}$ \\ Departments of ${ }^{1}$ Glaucoma, ${ }^{2}$ Fundus Disease and ${ }^{3}$ Cataract Surgery, Hebei Eye Hospital, Xingtai, Hebei 054000, P.R. China
}

Received January 27, 2020; Accepted November 20, 2020

DOI: 10.3892/etm.2021.9683

\begin{abstract}
The present study aimed to explore the clinical role of heat shock protein 70 (HSP70) in patients with acute angle-closure glaucoma (AACG). Seventy-four AACG patients who were admitted to our hospital from April 2017 to April 2019 were enrolled as a study group (SG). A further 70 healthy people undergoing physical examinations during the same period were enrolled as a control group (CG). HSP70 concentration was compared between the two groups, and the clinical value of this protein in AACG was analyzed. HSP70 concentration in SG was significantly lower than that in $\mathrm{CG}$ $(\mathrm{P}<0.050)$. The sensitivity and specificity of HSP70 for diagnosing AACG were 79.73 and $74.29 \%$, respectively $(\mathrm{P}<0.001)$. HSP70 concentration was positively correlated with central anterior chamber depth and peripheral anterior chamber depth, but negatively correlated with anterior angle and intraocular pressure $(\mathrm{P}<0.001)$. HSP70 had a relatively satisfactory predictive value for adverse reactions during the treatment $(\mathrm{P}<0.001)$. HSP70 concentration was markedly reduced in AACG patients, and its detection had a relatively satisfactory predictive value for AACG. Thus, HSP70 may be a potential and notable indicator for diagnosing and treating glaucoma in the future.
\end{abstract}

\section{Introduction}

As a clinical ophthalmic disease that has an extremely high incidence and causes great harm, glaucoma causes severe damage to tissues of various parts in the eyeball due to the imbalance of intraocular pressure (IOP) $(1,2)$. According to Garway-Heath et al, more than 900,000 people newly developed glaucoma worldwide in 2015, and the disease may occur in people of all ages (3). According to Wang et al, the incidence of glaucoma has increased by approximately

Correspondence to: Dr Hong Chen, Department of Glaucoma, Hebei Eye Hospital, 399 Quanbei East Street, Xingtai, Hebei 054000, P.R. China

E-mail: hf64bf@163.com

Key words: heat shock protein 70, acute angle-closure glaucoma, adverse reactions, prediction 10-fold compared with that 10 years ago. Thus, the incidence is predicted to continue to gradually increase, which makes glaucoma a most significant and daunting problem in clinical ophthalmology (4). Glaucoma patients usually suffer from optic atrophy and visual deterioration, and approximately $70 \%$ of them will go blind if they are not treated in a proper or timely manner $(5,6)$. Li et al have predicted that by 2020 , approximately 20 million people worldwide will develop blindness caused by glaucoma (7). Acute angle-closure glaucoma (AACG) is an eye disease in which the IOP undergoes a sharp increase due to the sudden closure of the anterior chamber angle. As the most serious type of glaucoma, AACG progresses to complete loss of sight within 24 to $48 \mathrm{~h}$ if not diagnosed and treated in time (8). The disease has no obvious and special symptoms, and some patients with strong pain tolerance experience only eye discomfort, and this may lead to loss of optimal treatment timing (9). At present, diagnostic methods for AACG are complex, therefore the disease requires evaluation and detection of various aspects (10), which is not conducive to its treatment because it has an extremely fast onset. AACG is relatively complex in clinical practice, and it is impossible to carry out large-scale clinical surveys and early screening. As the challenges of glaucoma become more serious, it is urgent to find an effective serum marker for the early screening or auxiliary diagnosis of AACG.

Highly conserved in structure, heat shock proteins (HSP) are a family of proteins that are produced by prokaryocytes and eukaryocytes under high temperature or other stresses (11). HSP70 that can inhibit apoptosis is widely distributed in all tissues and structures except photoreceptors (11). According to a previous study, it is a crucial gene locus for eye development, and is aberrantly expressed in glaucoma (12). However, there is currently little in-depth research on this protein in AACG. Therefore, whether this protein has potential as a reliable marker for diagnosing and treating AACG in the future remains to be confirmed. Thus, it is of great significance to conduct research on HSP70 at home and abroad to improve its clinical value in glaucoma and to confirm its effects on AACG.

\section{Materials and methods}

General information. A prospective analysis was performed on 74 AACG patients [enrolled as the study group (SG)] who were admitted to Hebei Eye Hospital (Xingtai, China) from 
April 2017 to April 2019 and on 70 healthy people [enrolled as the control group (CG)] who underwent physical examinations during the same period. The present study was approved by the Ethics Committee of Hebei Eye Hospital. All research subjects signed an informed consent form. General characteristics of all participants are listed in Table I.

Inclusion and exclusion criteria. The inclusion criteria were as follows: i) patients whose clinical manifestations were consistent with glaucoma symptoms, and who were diagnosed with AACG at our hospital; ii) patients who were operated on after diagnosis; iii) patients aged 20-60 years; and iv) patients with complete medical records. Exclusion criteria were as follows: i) patients complicated with other eye diseases; ii) iii) patients with severe cardiovascular and cerebrovascular diseases; iv) patients with tumors; v) patients complicated with other congenital immunodeficiency diseases or infectious diseases; vi) patients with mental disorders; vii) patients complicated with diabetes mellitus; viii) patients with physical disabilities; ix) patients with a contraindication to surgery; and x) patients who had transferred to other hospitals.

Methods. Surgery for the patients was performed by senior ophthalmologists at our hospital. Conventional treatment was as follows: YAG laser peripheral iridectomy was conducted on patients in the prodromal and intermittent stages. Positive rescue was conducted on patients in the acute attack stage, and the anterior chamber was opened as soon as possible to prevent permanent goniosynechia. Drugs (alternative drugs included $2 \%$ pilocarpine solution; acetazolamide; glycerol; $2 \%$ lidocaine; $20 \%$ mannitol) were first used to reduce IOP, and then the patients were operated after their blood pressure lowered and their congestive inflammation subsided. According to the IOP and anterior chamber, filtration surgery or peripheral iridectomy was selected, and the eyeball would be removed if necessary. Fasting venous blood $(4 \mathrm{ml})$ was respectively drawn from the patients before and after the treatment, allowed to stand at room temperature for $30 \mathrm{~min}$, and then centrifuged for $10 \mathrm{~min}\left(1,809 \mathrm{x} \mathrm{g}\right.$, at $\left.4^{\circ} \mathrm{C}\right)$, to obtain the upper serum. The concentration of HSP70 in the serum was detected by enzyme-linked immunosorbent assay (ELISA), and the kit (cat. no. JLC11484) was purchased from Shanghai Jingkang Bioengineering Co., Ltd., with the steps strictly and sterilely carried out according to the instructions of the kit.

Outcome measures. The concentration of serum HSP70 in the two groups; the predictive value of HSP70 for AACG; the correlation of pre-treatment HSP70 with IOP, central anterior chamber depth (CACD), peripheral anterior chamber depth (PACD) and anterior angle (AA); the predictive value of HSP70 for adverse reactions during treatment; changes of HSP70 concentration before and after treatment in SG.

Statistical methods. SPSS24.0 (IBM Corp.) was used to analyze and process the data. GraphPad 8 (GraphPad Software, Inc.) was used to plot figures. Count data were expressed by (rate), and Chi-square test was used for their comparison between groups. Measurement data were expressed by the mean \pm standard deviation (SD), and independent sample t-test was used for their comparison between groups. Spearman Correlation
Coefficient was used for correlation analysis. Receiver operating characteristic (ROC) curves were plotted to analyze the predictive value of HSP70. $\mathrm{P}<0.05$ indicated a statistically significant difference.

\section{Results}

Comparison of general information. There were no significant differences between the SG and CG in terms of age, body mass index (BMI), blood pressure, heart rate, sex, dwelling environment, educational background, smoking and alcohol drinking habits $(\mathrm{P}>0.050$; Table I).

Comparison of HSP70 concentration. The concentration of HSP70 in the SG was significantly lower than that in the CG before treatment $(\mathrm{P}<0.05)$. According to the ROC curve, when the cut-off value was 6.27, the sensitivity of HSP70 for diagnosing AACG was $79.73 \%$, the specificity was $74.29 \%$, the area under the curve (AUC) was 0.824 , and the $95 \%$ confidence interval (CI) was 0.757-0.891 ( $\mathrm{P}<0.001$; Fig. 1).

Correlation of HSP70 with indicators related to the course of the disease. According to the Pearson correlation coefficient, the concentration of HSP70 in the SG was positively correlated with CACD $(r=0.597, \mathrm{P}<0.001)$ and PACD $(\mathrm{r}=0.649, \mathrm{P}<0.001)$, but negatively correlated with AA $(\mathrm{r}=-0.467, \mathrm{P}<0.001)$ and IOP (r=-0.745, P<0.001) (Fig. 2).

Predictive value of HSP70 for adverse reactions. During treatment of all patients, there were 5 cases of fibrinoid exudation, 3 cases of anterior chamber inflammation, 3 cases of choroidal detachment, 4 cases of corneal edema and 2 cases of age-related macular degeneration, with an incidence of adverse reactions at $22.97 \%$. Patients who developed adverse reactions were considered as Group A $(n=17)$, while those without adverse reactions were considered as Group B $(n=57)$. The concentration of HSP70 in Group A was significantly lower than that in Group B $(\mathrm{P}<0.05)$. According to the ROC curve, when the cut-off value was 5.815 , the sensitivity of HSP70 for diagnosing AACG was $94.12 \%$, the specificity was $61.40 \%$, the AUC was 0.813, and the 95\% CI was 0.717-0.909 $(\mathrm{P}<0.001$; Fig. 3).

Changes of HSP70 concentration before and after treatment. In the SG, the concentration of HSP70 after treatment was significantly higher than that before treatment ( $\mathrm{P}<0.05$; Fig. 4).

\section{Discussion}

In modern society, the incidence of eye diseases has been annually increasing as the population ages (13). As the most serious type of glaucoma, AACG has no obvious symptoms in its early stage, and causes great damage to the eyeball and surrounding tissues by increasing IOP. Therefore, the improper or untimely treatment of the disease easily causes irreversible damage to the visual system of the patient $(14,15)$. Therefore, finding effective examination indices to increase the early diagnostic rate of AACG is the focus and difficulty of clinical research (16). In the present study, through the investigation of HSP70 in the blood of AACG patients, it was confirmed 
Table I. Comparison of general information [n (\%)].

\begin{tabular}{|c|c|c|c|c|}
\hline Features & $S G(n=74)$ & $\mathrm{CG}(\mathrm{n}=70)$ & $\chi^{2}$ or $\mathrm{t}$ & P-value \\
\hline Age (years) & $52.8 \pm 10.4$ & $53.6 \pm 11.6$ & 0.436 & 0.663 \\
\hline BMI $\left(\mathrm{kg} / \mathrm{cm}^{2}\right)$ & $22.62 \pm 3.54$ & $22.86 \pm 3.84$ & 0.390 & 0.697 \\
\hline Systolic blood pressure (mmHg) & $116.62 \pm 10.98$ & $117.24 \pm 11.24$ & 0.335 & 0.738 \\
\hline Diastolic blood pressure $(\mathrm{mmHg})$ & $74.62 \pm 7.85$ & $75.14 \pm 8.06$ & 0.392 & 0.696 \\
\hline Sex & & & 0.355 & 0.551 \\
\hline Male & $30(40.54)$ & $25(35.71)$ & & \\
\hline Female & $44(59.46)$ & $45(64.29)$ & & \\
\hline Dwelling environment & & & 0.716 & 0.397 \\
\hline City & $65(87.84)$ & $58(82.86)$ & & \\
\hline Countryside & $9(12.16)$ & $12(17.14)$ & & \\
\hline Educational background & & & 0.298 & 0.585 \\
\hline$<$ Senior high school & $34(45.95)$ & $29(41.43)$ & & \\
\hline$\geq$ Senior high school & $40(54.05)$ & $41(58.57)$ & & \\
\hline Smoking & & & 0.598 & 0.440 \\
\hline Yes & $25(33.78)$ & $28(40.00)$ & & \\
\hline No & $49(66.22)$ & $42(60.00)$ & & \\
\hline Alcohol drinking & & & 0.337 & 0.561 \\
\hline Yes & $20(27.03)$ & $22(31.43)$ & & \\
\hline No & $54(72.97)$ & $48(68.57)$ & & \\
\hline
\end{tabular}

SG, study group; CG, control group; BMI, body mass index.
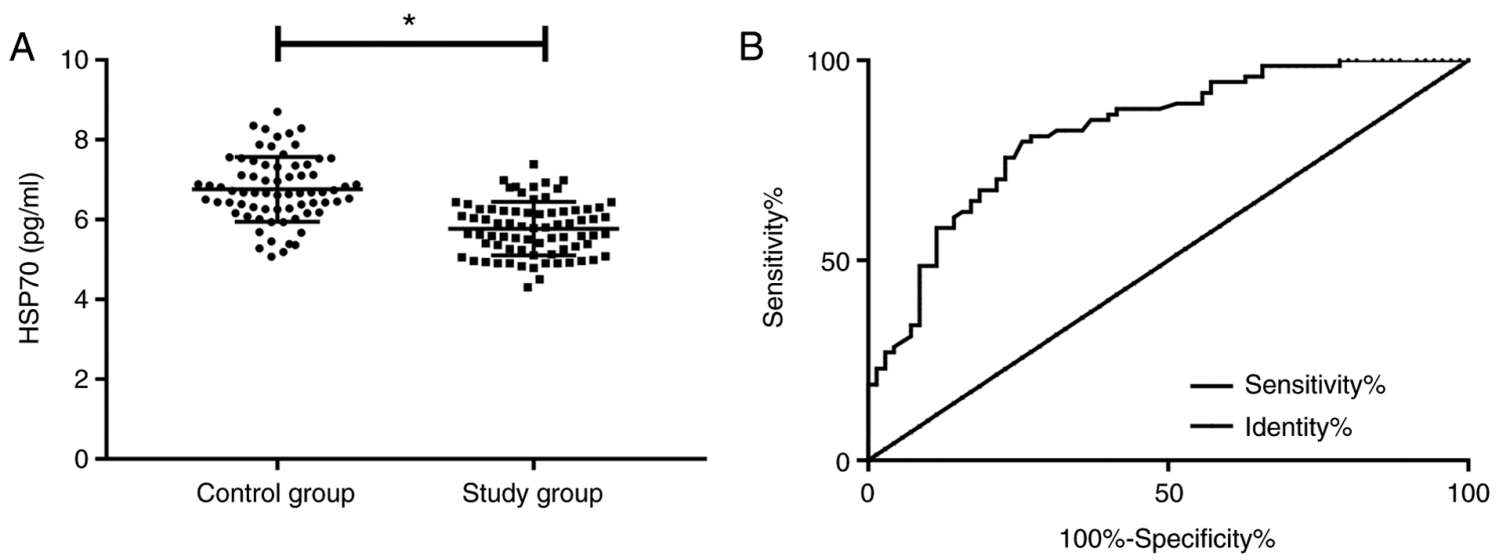

Figure 1. Comparison of HSP70 concentration and diagnostic value of HSP70. (A) The concentration of HSP70 in the SG was significantly lower than that in the CG. "P<0.050. (B) Analysis of the ROC curve of HSP70 for diagnosing AACG. HSP70, heat shock protein 70; SG, study group; CG, control group; ROC, receiver operating characteristic; AACG, acute angle-closure glaucoma.

through experimental analysis whether HSP70 has the potential to be a diagnostic and therapeutic marker for AACG, in order to provide a reliable theoretical basis for conducting large-scale and early screening and for assessing the progression of the disease in the future.

In the present study, HSP70 concentration was significantly reduced in the serum of the patients, suggesting that this protein may be involved in the development or progression of AACG. Waugh et al also studied HSP70 in patients with eye diseases (17), and their findings are consistent with the present experimental results and support our conclusions. The aforementioned study detected the expression of multiple genes and proteins in glaucoma, and determined that HSP70 was aberrantly expressed in the disease. In the present research, the expression of HSP70 in AACG was analyzed. Compared with previous studies, our research is more detailed on the types of targeted diseases, which can greatly reduce the contingency of experimental results. According to previous studies, the development of glaucoma is closely related to the death of retinal ganglion cells, and the death mechanism is associated with the increase in the apoptotic rate. The increase can be caused by blocked 

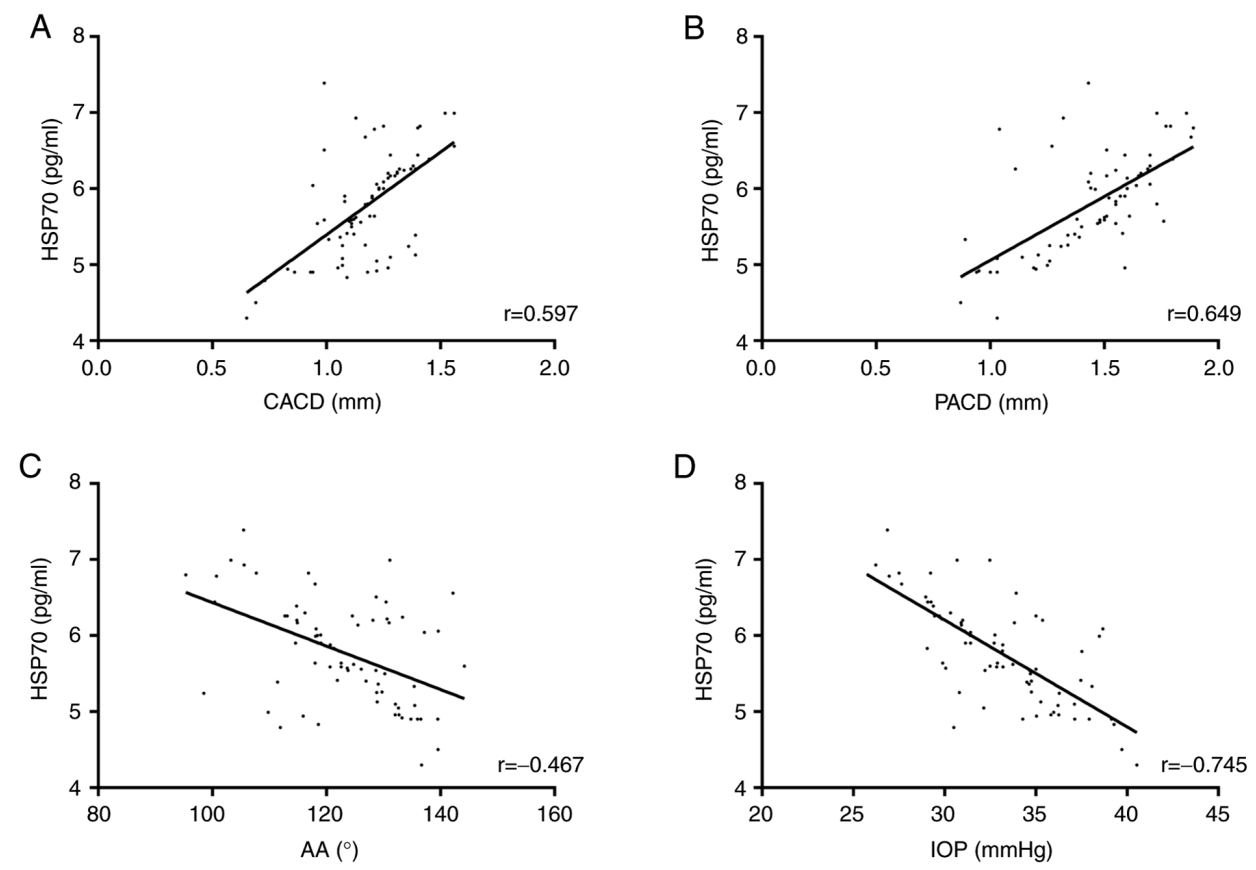

Figure 2. Correlation between HSP70 and indicators related to course of disease. (A) The correlation between HSP70 and CACD. (B) The correlation between HSP70 and PACD. (C) The correlation between HSP70 and AA. (D) The correlation between HSP70 and IOP. HSP70, heat shock protein 70; CACD, central anterior chamber depth; PACD, peripheral anterior chamber depth; AA, anterior angle; IOP, intraocular pressure.
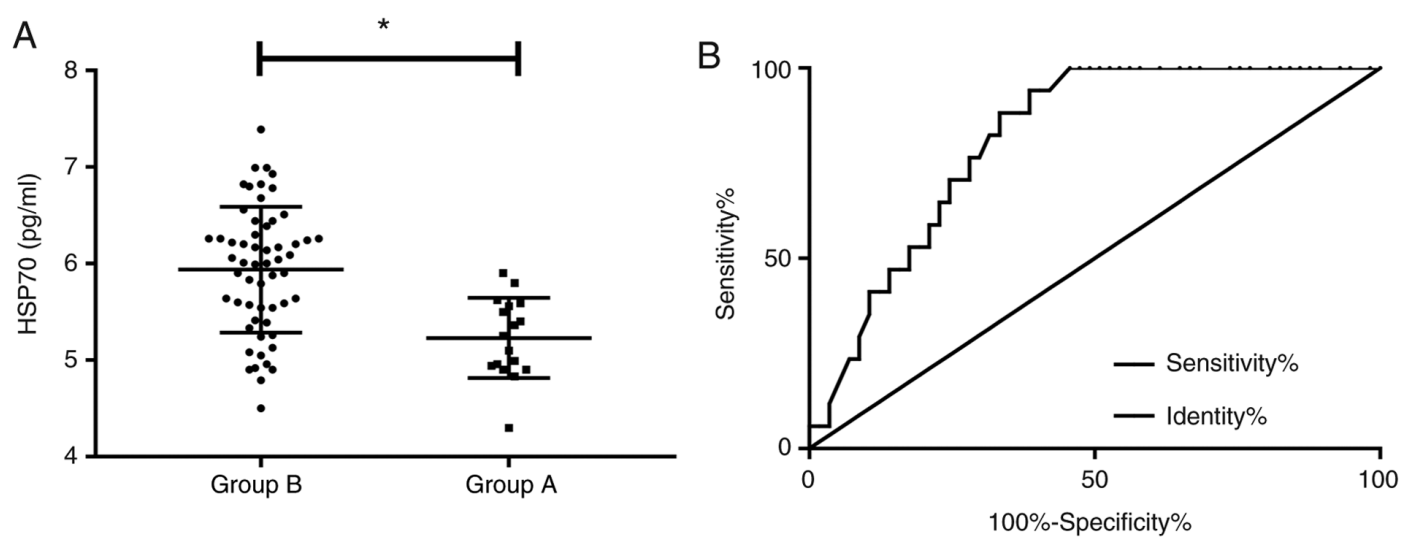

Figure 3. Predictive value of HSP70 for adverse reactions. (A) The concentration of HSP70 in Group A (patients who developed adverse reactions) was significantly lower than that in Group B (patients who did not develop adverse reactions) " $\mathrm{P}<0.05$. (B) ROC curve analysis of HSP70 on adverse reactions. HSP70, heat shock protein 70; ROC, receiver operating characteristic.

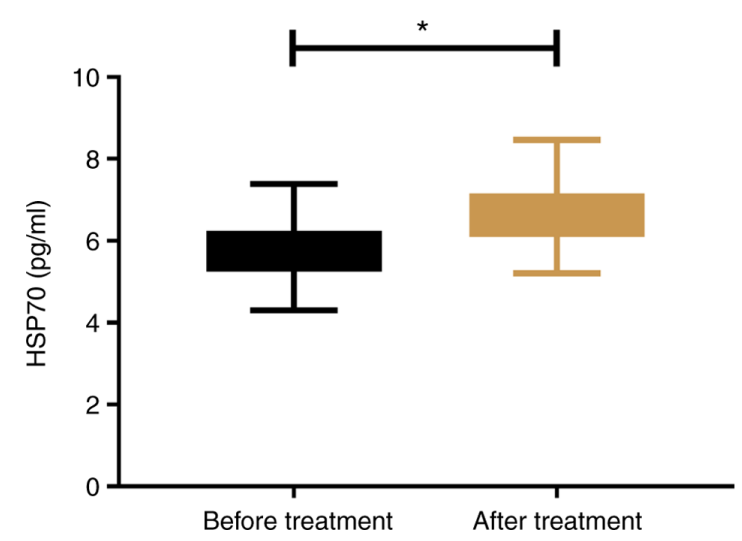

Figure 4. Changes of HSP70 concentration before and after treatment. In the SG, the concentration of HSP70 after treatment was significantly higher than that before treatment. ${ }^{*} \mathrm{P}<0.001$. HSP70, heat shock protein $70 ; \mathrm{SG}$, study group. retrograde transport, lack of nutritional factors and excitatory amino acids (18-20). HSP70 has an anti-apoptotic effect on cells, and induces HSP to produce endogenous protection in neuronal cells (21). One of its forms of gene induction is HSPA1B (22), which is a risk factor for glaucoma according to Salehi et al (23). Therefore, it is inferred that the mechanism of action of HSP70 in AACG may be correlated with its differentiation into HSPA1B. However, since basic experiments were not carried out, it is impossible to determine the exact mechanism of action of HSP70 on AACG, which will be fully investigated in future research. According to the ROC curve, the sensitivity and specificity of HSP70 for predicting AACG were 79.79 and $74.29 \%$, respectively, indicating that this protein can be used as a clinical screening index that may aid doctors in diagnosing AACG in the future. Serum markers are superior to conventional diagnostic methods for AACG in 
terms of the convenient acquisition of test samples, the longer storage time of blood samples, and convenient reexamination. Moreover, the detection results of the markers are objective and do not rely on human subjective consciousness to assess the disease, which reduces man-made misdiagnosis or missed diagnosis. In the present study, the concentration of HSP70 was significantly correlated with IOP, CACD, PACD and AA. This further confirms the close relationship between HSP70 and the progression of AACG, and is similar to the inference aforementioned. AACG is a retinopathy during whose progression the normal activity of retinal neuron cells plays an important role (24). HSP70 is a protective factor of neuronal cells. The occurrence of AACG greatly reduces it and then aggravates neuronal damage. As a result, the disease more markedly deteriorates. According to Wang et al, the lesion of AACG is closely related to neuronal damage (25). In the present study, HSP70 had a relatively satisfactory predictive value for adverse reactions during treatment, and its concentration significantly increased after treatment, which demonstrated that HSP70 may be used for the diagnosis and treatment of AACG in the future. In addition, if the mechanism of HSP70 on the protection of neuronal cells and the relationship between HSP70 and glaucoma are fully elucidated, then, HSP70 may become a therapeutic target for glaucoma and even various eye diseases in the future, thus rendering it a great scientific application prospect.

The purpose of the present study was to explore the effects of HSP70 on AACG patients. Previous studies have suggested that HSP and glaucoma are related (26-28), but the present study focused on the analysis of AACG in glaucoma and explored the significance of HSP as a diagnostic marker in AACG, to the best of our knowledge, for the first time. However, there are still numerous shortcomings due to the limited experimental conditions. For example, the exact mechanism of action of HSP70 on AACG was not determined due to the failure to conduct basic experiments. The effects of HSP70 on the long-term prognosis of patients remain unclear due to the short experimental period. In addition, the present study focused and analyzed mainly AACG, thus HSP70 may be aberrantly expressed in other pathological types of glaucoma, yet to be determined. In the present study, HSP70 was only detected in the blood of the patients, and its expression has not been evaluated in glaucoma-related cells. In the future, more detailed and in-depth experiments, analyses and discussions will be conducted, to address these deficiencies and obtain more definitive results.

In summary, HSP70 concentration was markedly reduced in AACG patients, and its detection had a relatively satisfactory predictive value for AACG, and thus HSP70 may be a potential and effective indicator for diagnosing and treating glaucoma in the future.

\section{Acknowledgements}

Not applicable.

\section{Funding}

No funding was received.

\section{Availability of data and materials}

The datasets used and/or analyzed during the current study are available from the corresponding author on reasonable request.

\section{Authors' contributions}

HC wrote the manuscript. HC, AT and YW conceived and designed the study. RL and $\mathrm{RH}$ were responsible for the collection and analysis of the experimental data. XX and SC interpreted the data and drafted the manuscript. HC and AT revised the manuscript critically for important intellectual content. All authors read and approved the final manuscript.

\section{Ethics approval and consent to participate}

The present study was approved by the Ethics Committee of Hebei Eye Hospital (Xingtai, China). Patients who participated in this research, signed the informed consent and had complete clinical data. Signed written informed consents were obtained from the patients and/or guardians.

\section{Patient consent for publication}

Not applicable.

\section{Competing interests}

The authors declare that they have no competing interests.

\section{References}

1. Liu L, Jia Y, Takusagawa HL, Pechauer AD, Edmunds B, Lombardi L, Davis E, Morrison JC and Huang D: Optical coherence tomography angiography of the peripapillary retina in glaucoma. JAMA Ophthalmol 133: 1045-1052, 2015.

2. Haffner DS, Smedley GT, Tu H and Burns TW: Devices and methods for glaucoma treatment: U.S. Patent 9,597,230. Filed August 8, 2007; issued March 21, 2017.

3. Garway-Heath DF, Crabb DP, Bunce C, Lascaratos G, Amalfitano F, Anand N, Azuara-Blanco A, Bourne RR, Broadway DC, Cunliffe IA, et al: Latanoprost for open-angle glaucoma (UKGTS): A randomised, multicentre, placebo-controlled trial. Lancet 385: 1295-1304, 2015.

4. Wang X, Jiang C, Ko T, Kong X, Yu X, Min W, Shi G and Sun X: Correlation between optic disc perfusion and glaucomatous severity in patients with open-angle glaucoma: An optical coherence tomography angiography study. Graefes Arch Clin Exp Ophthalmol 253: 1557-1564, 2015.

5. Yarmohammadi A, Zangwill LM, Diniz-Filho A, Suh MH, Manalastas PI, Fatehee N, Yousefi S, Belghith A, Saunders LJ, Medeiros FA, et al: Optical coherence tomography angiography vessel density in healthy, glaucoma suspect, and glaucoma eyes. Invest Ophthalmol Vis Sci 57: OCT451-OCT459, 2016.

6. Pfeiffer N, Garcia-Feijoo J, Martinez-De-La-Casa JM, Larrosa JM, Fea A, Lemij H, Gandolfi S, Schwenn O, Lorenz K and Samuelson TW: A randomized trial of a Schlemm's canal microstent with phacoemulsification for reducing intraocular pressure in open-angle glaucoma. Ophthalmology 122: 1283-1293, 2015.

7. Li Z, Allingham RR, Nakano M, Jia L, Chen Y, Ikeda Y, Mani B, Chen LJ, Kee C, Garway-Heath DF, et al: A common variant near TGFBR3 is associated with primary open angle glaucoma. Hum Mol Genet 24: 3880-3892, 2015.

8. Lai J, Choy BN and Shum JW: Management of primary angle-closure glaucoma. Asia Pac J Ophthalmol (Phila) 5: 59-62, 2016.

9. Bansal S, Balakrishnan SA, Blachley T, Weizer JS, Lee PP and Stein JD: Subsequent receipt of interventions for glaucoma among a nationwide sample of patients who underwent laser peripheral iridotomy. Am J Ophthalmol 160: 275-282.e4, 2015. 
10. Wright C, Tawfik MA, Waisbourd M and Katz LJ: Primary angle-closure glaucoma: An update. Acta Ophthalmol 94: 217-225, 2016

11. Shevtsov M and Multhoff G: Heat shock protein-peptide and HSP-based immunotherapies for the treatment of cancer. Front Immunol 7: 171, 2016.

12. Shi H, Zhang J, Zhu R, Hu N, Lu H, Yang M, Qin B, Shi J and Guan H: Primary angle closure and sequence variants within MicroRNA binding sites of genes involved in eye development. PLoS One 11: e0166055, 2016.

13. Uhr JH, Mishra K, Wei C and Wu AY: Awareness and knowledge of emergent ophthalmic disease among patients in an internal medicine clinic. JAMA Ophthalmol 134: 424-431, 2016.

14. Stegmann RC, Grieshaber MC and Grieshaber HR: Method and device for the treatment of glaucoma: U.S. Patent 8,951,221. Filed August 20, 2009; issued February 10, 2015.

15. Lévêque PM, Zéboulon P, Brasnu E, Baudouin C and Labbé A: Optic disc vascularization in glaucoma: Value of spectral-domain optical coherence tomography angiography. J Ophthalmol 2016: 6956717, 2016

16. Waisbourd M, Pruzan NL, Johnson D, Ugorets A, Crews JE, Saaddine JB, Henderer JD, Hark LA and Katz LJ: The Philadelphia glaucoma detection and treatment project: Detection rates and initial management. Ophthalmology 123: 1667-1674, 2016.

17. Waugh DT: The contribution of fluoride to the pathogenesis of eye diseases: Molecular mechanisms and implications for public health. Int J Environ Res Public Health 16: 856, 2019.

18. Li CP, Wang SH, Wang WQ, Song SG and Liu XM: Long noncoding RNA-Sox2OT knockdown alleviates diabetes mellitus-induced retinal ganglion cell (RGC) injury. Cell Mol Neurobiol 37: 361-369, 2017

19. Yadav KS, Sharma S and Londhe VY: Bio-tactics for neuroprotection of retinal ganglion cells in the treatment of glaucoma. Life Sci 243: 117303, 2020.
20. Almasieh M, Wilson AM, Morquette B, Cueva Vargas JL and Di Polo A: The molecular basis of retinal ganglion cell death in glaucoma. Prog Retin Eye Res 31: 152-181, 2012.

21. Zhang MH, Zhou XM, Cui JZ, Wang KJ, Feng Y and Zhang HA: Neuroprotective effects of dexmedetomidine on traumatic brain injury: Involvement of neuronal apoptosis and HSP70 expression. Mol Med Rep 17: 8079-8086, 2018.

22. Fang CT, Kuo HH, Pan TS, Yu FC and Yih LH: HSP70 regulates the function of mitotic centrosomes. Cell Mol Life Sci 73: 3949-3960, 2016.

23. Salehi Z, Gholaminia M, Gholaminia Z, Panjtanpanah M and Qazvini MG: The GG genotype of the HSPA1B gene is associated with increased risk of glaucoma in northern Iran. Mol Biol (Mosk) 51: 31-36, 2017 (In Russian).

24. Della Santina L and Ou Y: Who's lost first? Susceptibility of retinal ganglion cell types in experimental glaucoma. Exp Eye Res 158: 43-50, 2017.

25. Wang X, Jiang C, Kong X, Yu X and Sun X: Peripapillary retinal vessel density in eyes with acute primary angle closure: An optical coherence tomography angiography study. Graefes Arch Clin Exp Ophthalmol 255: 1013-1018, 2017.

26. Ayub H, Khan MI, Micheal S, Akhtar F, Ajmal M, Shafique S, Ali SH, den Hollander AI, Ahmed A and Qamar R: Association of eNOS and HSP70 gene polymorphisms with glaucoma in Pakistani cohorts. Mol Vis 6: 18-25, 2010.

27. Nowak A, Majsterek I,Przybyłowska-SygutK,PytelD,Szymanek K, Szaflik J and Szaflik JP: Analysis of the expression and polymorphism of APOE, HSP, BDNF, and GRIN2B genes associated with the neurodegeneration process in the pathogenesis of primary open angle glaucoma. Biomed Res Int 2015: 258281, 2015.

28. Nowak A,SzaflikJP,Gacek M,Przybylowska-Sygut K, Kamińska A, Szaflik J and Majsterek I: BDNF and HSP gene polymorphisms and their influence on the progression of primary open-angle glaucoma in a Polish population. Arch Med Sci 10: 1206-1213, 2014. 